[Article]

\title{
氧化石墨烯在氧化锌祄底上的电化学还原及其光电性能
}

\author{
李一鸣 ${ }^{1}$ 陈 肖 ${ }^{1}$ 刘晓军 ${ }^{1}$ 李文有 ${ }^{1}$ 贺蕴秋 ${ }^{1,2, *}$ \\ ('同济大学材料科学与工程学院, 上海 201804; 2 先进土木工程材料教育部重点实验室, 上海 201804)
}

\begin{abstract}
摘要: 采用阳极电泳法, 在氧化锌 $(\mathrm{ZnO})$ 祄底上沉积氧化石墨烯(GO) 以形成 GO-ZnO 双层复合膜; 采用阴极 恒电位法, 对复合膜上的 GO 进行还原。对不同还原时间的 GO, 通过X射线光电子能谱(XPS), 傅里叶变换 红外(FTIR)光谱, 场发射扫描电子显微镜(FESEM)等手段对其结构变化进行表征, 采用紫外-可见(UV-Vis)分 光光度法和电化学测试手段对其能级演变进行考察, 并对两者的对应关系进行了讨论。研究发现, 当 $\mathrm{GO}$ 膜 达到最大还原态后, 随还原时间增加还会出现进一步的结构转变, 并最终碎裂生成边缘羧基增多的小尺寸 GO。GO 能隙均减小至可见光范围, 其能级位置及半导体极性也产生了不同的改变。由对复合膜的光电化学

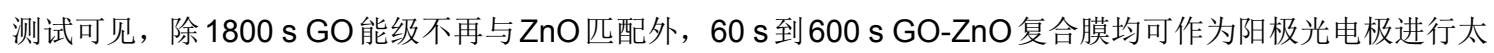
阳光电转换。对光电性能差异的讨论则可得, GO 膜碎裂造成叠层形貌向无序形貌的转变有利于光电转换性 能的提升。
\end{abstract}

关键词: 氧化石墨烯-氧化锌复合膜; 电化学还原; 结构转变; 能级演变; 光电转换 中图分类号: 0646

\section{Electrochemical Reduction of Graphene Oxide on ZnO Substrate and Its Photoelectric Properties}

\author{
LI Yi-Ming ${ }^{1}$ CHEN Xiao ${ }^{1} \quad$ LIU Xiao-Jun ${ }^{1} \quad$ LI Wen-You $^{1} \quad$ HE Yun-Qiu ${ }^{1,2, *}$ \\ ( ${ }^{1}$ School of Materials Science and Engineering, Tongji University, Shanghai 201804, P. R. China; \\ ${ }^{2}$ Key Laboratory of Advanced Civil Engineering Materials, Ministry of Education, Shanghai 201804, P. R. China)
}

\begin{abstract}
Abstrct: In the present work, graphene oxide (GO)-ZnO bilayer composites were fabricated by depositing $\mathrm{GO}$ on $\mathrm{ZnO}$ by an anodic electrophoretic method. The composite films were then subjected to a cathodic electrochemical treatment with different $\mathrm{GO}$ reduction times. The as-prepared films were characterized by $X-$ ray photoelectron spectroscopy (XPS), Fourier transform infrared (FTIR) spectroscopy and field emission scanning electron microscopy (FESEM) to study changes in the GO structure. The evolution of the material's energy levels over time was also determined by ultraviolet-visible (UV-Vis) spectroscopy and electrochemical measurements. A series of structural transformations of $\mathrm{GO}$ occurred even after it had reached the maximum degree of reduction. Prolonged treatment saw the $G O$ flakes fracture into smaller $G O$ particles with a sharp increase in the proportion of carboxyl groups. The energy gap of $\mathrm{GO}$ varied and extended into the visible range with longer reduction time. The energy levels and charge carrier type also varied. Photoelectrochemical tests on the samples revealed that the 60 to 600 -s reduced GO-ZnO composite films showed photoelectric conversion behavior as photoanodes. However, the sample reduced for $1800 \mathrm{~s}$ was not effective at light-harvesting owing to lowering of the $\mathrm{GO}$ conduction band below that of $\mathrm{ZnO}$. The differences in performance indicated that the transformation of the laminated GO geometry to a more disordered distribution enhanced conversion efficiency.
\end{abstract}

Received: August 29, 2016; Revised: November 16, 2016; Published online: November 17, 2016.

*Corresponding author. Email: heyunqiu@tongji.edu.cn.

The project was supported by the National Natural Science Foundation of China (51172162).

国家自然科学基金(51172162)资助项目

(c) Editorial office of Acta Physico-Chimica Sinica 
Key words: Graphene oxide-ZnO composite film; Electrochemical reduction; Structural transformation; Energy level evolution; Photoelectric conversion

\section{1 引言}

作为制备石墨烯粉体的常见前驱物, 氧化石 墨烯(GO)在近年来受到愈加广泛的关注。GO结构 中吸附在六方蜂窝碳原子网络上的大量氧官能团 使基面晶格产生畸变，从而使 GO 具有不同于石墨 烯的独特光学和电学性质 1 。对 GO 能级结构的理 论和实验研究均指出, 不同还原程度 $\mathrm{GO}$ 的能隙宽 度会出现改变 ${ }^{2-4}$ 。利用此特性, 通过对 GO 进行不 同程度的还原, 可使其在一系列光电领域, 如荧 光 ${ }^{5}$ 光探测 6,7 、光催化 ${ }^{8,9}$ 等领域具有应用潜力。当 前对 GO 还原方法的诸多研究可主要归纳为三类, 即化学法 ${ }^{10,11}$ 、热处理法 ${ }^{10}$ 和电化学法 ${ }^{10,12}$ 。其中, 化学法对 $\mathrm{GO}$ 的还原通常会引起 $\mathrm{GO}$ 晶格的杂质掺 杂, 而热处理则通常会造成 $G O$ 晶格空位缺陷的增 多 ${ }^{10}$ 。电化学法不会向 GO 引入杂质, 还原条件较 为温和。由于其对 $\mathrm{GO}$ 的还原通常需要使 $\mathrm{GO}$ 成 膜, 因而尤其适用于对薄膜 $\mathrm{GO}$ 还原的情况。

我们在之前的研究 ${ }^{13}$ 中采用恒电位法对沉积在 掺氟 $\mathrm{SnO}_{2}(\mathrm{FTO})$ 衬底上的 $\mathrm{GO}$ 薄膜进行还原, 并验 证了适当还原的 $\mathrm{GO}$ 作为可见光吸收体进行太阳光 电转换的可行性。为构造光电极, 通常需要将适 当还原的 GO 与金属氧化物复合形成异质结。在相 关研究中, Chen 等 ${ }^{14}$ 成功将 $\mathrm{GO}$ 与 $\mathrm{TiO}_{2}$ 复合得到具 有可见光电转换能力的 $p-n$ 结。我们也曾报道不同 还原程度 $\mathrm{GO}$ 与 $\mathrm{ZnO}$ 的复合光电极具有不同的可见 光电转换性能 ${ }^{15}$ 。以上研究中的复合材料均为氧化 物颗粒包覆在无序分布的 $\mathrm{GO}$ 表面的混乱结构, 对 $\mathrm{GO}$ 还原程度进行调节的同时均会造成氧化物形貌 和结构的改变, 因此不能得到单一 $\mathrm{GO}$ 结构变化与 电极光电转换性能的对应关系。鉴于此, 一种解 决方案是通过分别制备金属氧化物层与 $\mathrm{GO}$ 层的双 层膜结构, 并对 GO进行原位电化学还原。

在本文中, 我们首先在 FTO 玻璃上沉积稀疏 的 $\mathrm{ZnO}$ 纳米柱阵列作为 $\mathrm{GO}$ 祄底, 并采用阳极电泳 法沉积 GO 以得到 GO- $\mathrm{ZnO}$ 双层复合膜。以有机溶 液为电解液, 以防止电极上质子的还原反应生成 氢气对复合膜结构的破坏 ${ }^{16}$, 并采用恒电位法对复 合膜中的 GO 进行不同时间的处理。本文对一系列 还原样品的化学结构和微观形貌进行了详细的表 征, 并着重讨论了对应能级结构及光电转换性能
的演变与以上结构变化的对应关系。研究证实了 在 $\mathrm{ZnO}$ 半导体与 $\mathrm{GO}$ 复合光电极中, 通过电化学法 对 GO进行原位结构调控的可行性。

\section{2 实验步骤与表征}

$\mathrm{ZnO}$ 纳米柱阵列采用两步电化学法制备。第 一步, 采用三电极体系, $\mathrm{FTO}$ 玻璃为工作电极, $\mathrm{Pt}$ 片和饱和甘录电极(SCE) 分别为对电极和参比电 极, 在恒电流条件 $\left(0.1 \mathrm{~mA} \cdot \mathrm{cm}^{-2}\right)$ 下从 $0.03 \mathrm{~mol} \cdot \mathrm{L}^{-1}$ $\mathrm{ZnCl}_{2}$ 的二甲基亚砜(DMSO)溶液中常温沉积 $150 \mathrm{~s}$ 得到 $\mathrm{ZnO}$ 种子层。第二步, 仍采用以上三电极体 系, 在恒电位 $-1 \mathrm{~V}$ 条件下从 $1 \mathrm{mmol} \cdot \mathrm{L}^{-1} \mathrm{Zn}\left(\mathrm{NO}_{3}\right)_{2}$ 和 $1 \mathrm{~mol} \cdot \mathrm{L}^{-1} \mathrm{NaNO}_{3}$ 的混合溶液 $\left(80^{\circ} \mathrm{C}\right.$ 水浴 $)$ 中沉积 $30 \mathrm{~min}$ 得到 $\mathrm{ZnO}$ 纳米柱层。以上沉积实验均采用 DJS-292 型恒电位仪(上海雷磁)进行。

$\mathrm{GO}$ 膜采用电泳法制备。具体地, 以 $\mathrm{ZnO}$ 纳米 柱薄膜为阳极 (有效面积为 $2 \mathrm{~cm} \times 2 \mathrm{~cm}$ ), 抛光后的 $316 \mathrm{~L}$ 不锈钢片为阴极, 电极间距为 $3 \mathrm{~mm}$ 。该两电 极体系在 $3 \mathrm{~V}$ 直流电压下从 $1 \mathrm{mg} \cdot \mathrm{mL}^{-1} \mathrm{GO}$ 的分散 液中沉积 $120 \mathrm{~s}$, 并在 $60^{\circ} \mathrm{C}$ 烘箱中烘干即得到 $\mathrm{GO}$ 膜样品。其中， GO 以 $400 \mathrm{~nm}$ 粒度的石墨 (99.95\%, 上海慈太龙化学试剂公司)为原料, 采用 改进的 Hummer 法 ${ }^{15}$ 制备。经超声分散后的新鲜 $\mathrm{GO}$ 溶液呈酸性, 在沉积前以 $1 \%(w)$ 的氨水调节其 $\mathrm{pH}$ 到 6 , 从而避免过酸的电泳液对 $\mathrm{ZnO}$ 结构的破 坏。以上沉积实验采用 DH1720A-1 型直流稳压稳 流电源(北京大华)进行。

在 GO 的电化学还原前, 将复合膜样品在 $105^{\circ} \mathrm{C}$ 烘箱中保温 $12 \mathrm{~h}$ 以最大程度脱去自由水, 从 而尽量避免电化学过程中的质子还原反应产生的 额外电流 ${ }^{17}$ 及水分子参与到 $\mathrm{GO}$ 的还原 ${ }^{18}$ 。 $\mathrm{GO}$ 的电 化学还原采用三电极体系, GO膜为阴极, $\mathrm{Pt}$ 片和 $\mathrm{Ag} / \mathrm{Ag}^{+}$电极分别为对电极和参比电极, 在 $0.1 \mathrm{~mol}$ $\mathrm{L}^{-1} \mathrm{LiClO}_{4}$ 的乙腈溶液( $\mathrm{Ar}$ 气饱和)中以恒电位 $(-2.1$ $\mathrm{V})$ 还原不同时间。最后, 将还原后的样品用乙醇 冲洗干净并于 $60^{\circ} \mathrm{C}$ 烘干以用作一系列结构表征和 性能测试。以上还原实验采用 $\mathrm{CHI} 660 \mathrm{C}$ 型电化学 工作站(上海辰华)上进行。

采用傅里叶变换红外光谱仪 (FTIR, Thermo Nicolet is5)和 X 射线光电子能谱仪 (XPS, Thermo 
ESCALAB 250Xi)对不同样品表面成分的化学结构 进行分析。场发射扫描电子显微镜(FESEM, Quanta $400 \mathrm{FEG}$ )和透射电子显微镜 (TEM, Hitachi H-800) 分别用来对样品的微观形貌特征和 $\mathrm{ZnO}$ 纳米柱的 结晶状态进行观察。紫外-可见分光光度计 (UVVis, Hitachi U-4100)被用来进行复合膜光透过性能 的对比, 通过对透过率曲线的计算 ${ }^{19}$ 得到 $\mathrm{GO}$ 的能 隙。各样品 GO 的能级结构可由不同的电化学测试 结果描述。样品的光电转换性能以瞬态光电流密 度响应作为指标。其中, 光电性能测试的激发光 源为一个太阳光强的 CEL-S500 型太阳光模拟器(北 京中教金源), 测试前在光源前覆盖紫外滤光片 (截 断波长为 $450 \mathrm{~nm}$ ) 以除去光谱中的紫外光。

在以上实验中, $\mathrm{Ag} / \mathrm{Ag}^{+}$电极为准参比电极, 它是通过将 $\mathrm{Ag}$ 丝插入装满 $0.01 \mathrm{~mol} \cdot \mathrm{L}^{-1} \mathrm{AgNO}_{3}$ 的 乙腈溶液的盐桥中得到的; 其电位经二茂铁溶液 校正 ${ }^{20}$ 后约为 $0.31 \mathrm{~V}(v s \mathrm{SCE})$ 。另外, 除特别说明 外, 以上各实验步骤所采用试剂均为 $\mathrm{AR}$ 纯度, 并 购自中国国药集团有限公司。

\section{3 结果与讨论}

对 $\mathrm{ZnO}$ 祄底, 由 FESEM 表征(图 S1(a)) (见 Supporting Information (SI)), 可见表面稀疏分布的 纳米柱, 且基本呈垂直取向。由 TEM 表征(图 S1 (b)), 可得纳米柱的半径分布不太均匀, 柱长约 300-500 nm。单个纳米柱的选区电子衍射(SAED) 斑点(图 S1(b)插图)归属于六方晶系, 从而表明 $\mathrm{ZnO}$ 纳米柱为结晶良好的六方单晶。向 $\mathrm{ZnO}$ 祄底 电泳 GO, 得到的 GO 薄膜具有规整的叠层形貌, 薄膜厚度约为 $80 \mathrm{~nm}$ (图 S1(c))。在电化学处理前对 $\mathrm{GO}-\mathrm{ZnO}$ 复合膜作循环伏安 $(\mathrm{CV})$ 测试, 得到首圈电 流密度随还原电位的变化曲线如图 1(a)所示。对首 段负向电流密度曲线的线性增长做切线, 得到电 流的起始电位约 $-1.66 \mathrm{~V}\left(v \mathrm{sg} / \mathrm{Ag}^{+}\right)$。对 FTO 和 $\mathrm{ZnO}$ 祄底作 $\mathrm{CV}$ 测试, 得到负向电流的起始电位分 别为 -2.25 和 $-2.49 \mathrm{~V}\left(v s \mathrm{Ag} / \mathrm{Ag}^{+}\right.$) (图 S2), 并分别与 $\mathrm{SnO}_{2}{ }^{12}$ 和电解质 (和/或溶剂) ${ }^{17}$ 的还原电位对应。鉴 于 FTO 与电解液较宽的电位窗口, $\mathrm{GO}-\mathrm{ZnO}$ 复合 膜电流的起始电位应与 $\mathrm{GO}$ 的还原电位对应, 即 为 $-1.66 \mathrm{~V}\left(v \mathrm{sg} / \mathrm{Ag}^{+}\right)$。文献 ${ }^{17}$ 指出, 通过适当增 加还原过电位, 可实现 $\mathrm{GO}$ 还原程度的提升。因 此, 我们采用略低于 $\mathrm{SnO}_{2}$ 还原电位的 $-2.1 \mathrm{~V}(\mathrm{vs}$ $\left.\mathrm{Ag} / \mathrm{Ag}^{+}\right)$进行电化学处理, 以期在不破坏祄底金属
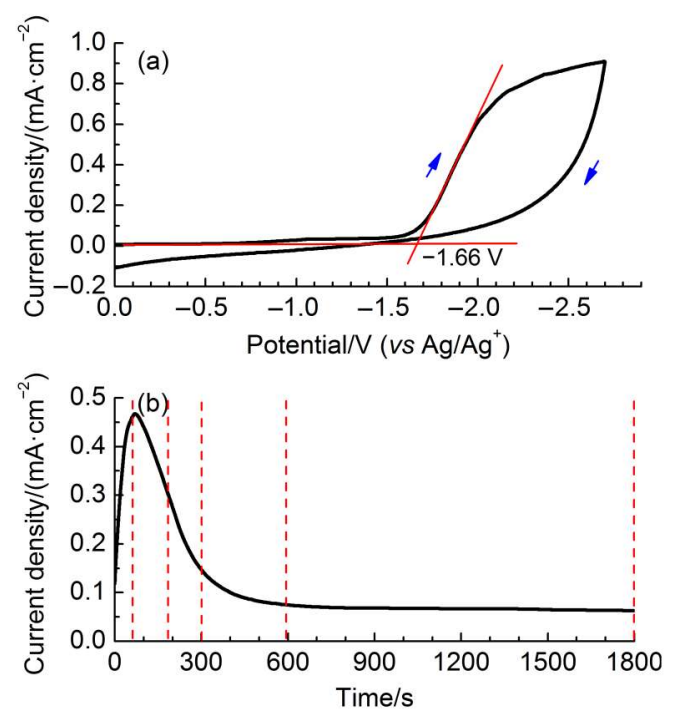

图 1 (a) GO-ZnO 复合膜的首圈循环伏安曲线(扫描速率 $\left.10 \mathrm{mV} \cdot \mathrm{s}^{-1}\right)$; (b) 复合膜在 $-2.1 \mathrm{~V}\left(\mathrm{vs} \mathrm{Ag} / \mathrm{Ag}^{+}\right)$恒电位条件下的 电流密度变化曲线

Fig.1 (a) The first circle of the cyclic voltammogram (CV) curve for the as-prepared GO-ZnO film with a scan rate of $10 \mathrm{mV} \cdot \mathrm{s}^{-1}$; (b) the current density-time curve during the electrochemical reduction of $\mathrm{GO}$ at $-2.1 \mathrm{~V}\left(\mathrm{vs} \mathrm{Ag} / \mathrm{Ag}^{+}\right)$

氧化物的前提下考察 $\mathrm{GO}$ 所能达到还原程度的最大 限度。图 1(b)为复合膜在电化学还原过程中的电流 密度随时间的变化曲线。电流密度随时间的增加 先增后减, 至 $600 \mathrm{~s}$ 后基本不变, 整体走势与文 献 ${ }^{17}$ 报道一致。文章选取 0、60、180、300、600 和 $1800 \mathrm{~s}$ 六组不同处理时间的复合膜样品, 以详细考 察样品结构在电流不同阶段的改变。

对不同处理时间 GO 的化学结构进行表征, 得 到复合膜 GO 表面 C $1 s$ XPS 拟合图谱如图 2(a)所 示, 在测试前对样品表面进行低能 $\mathrm{Ar}^{+}$束清洗以除 去各类碳杂质的影响。图中各样品对应的分峰结 合能及含量信息列于表 1 。由图可见, 随结合能的 增加, $\mathrm{C} 1 s$ 的拟合峰依次为 $\mathrm{C} s p^{2}$ 峰 $(284.4 \mathrm{eV}), \mathrm{C}$ $s p^{3}$ 及羟基 $\mathrm{C}-\mathrm{OH}$ 混合峰(约 $285.4 \mathrm{eV}$ ), 环氧基 $\mathrm{C}-$ $\mathrm{O}-\mathrm{C}$ 键(约 $286.5 \mathrm{eV}$ ), 羰基 $\mathrm{C}=\mathrm{O}$ 键 (约 $288.2 \mathrm{eV}$ ) 及羧基 $\mathrm{COOH}$ 峰(约 $289.8 \mathrm{eV})^{13,21}$ 。氧官能团相对含 量反映了 $\mathrm{GO}$ 的还原程度。同 $0 \mathrm{~s} \mathrm{GO}$ 相比, $60 \mathrm{~s}$ $\mathrm{GO}$ 各氧官能团含量大幅下降。其中, 由表 1 可见 “ $s p^{3}-\mathrm{C} \& \mathrm{C}-\mathrm{OH}$ ” 组分含量由 $11.51 \%$ 增加至 $17.98 \%$, 根据两样品表面 O $1 s$ XPS 的对比结果(图 $\mathrm{S} 3), 60 \mathrm{~s} \mathrm{GO}$ 中的羟基也大幅减少, 而氧官能团脱 去后生成的大量 $s p^{3}-\mathrm{C}$ 是该分峰增大的原因。因 此, $60 \mathrm{~s} \mathrm{GO}$ 较 $0 \mathrm{~s} \mathrm{GO}$ 产生了较大幅度的还原。 

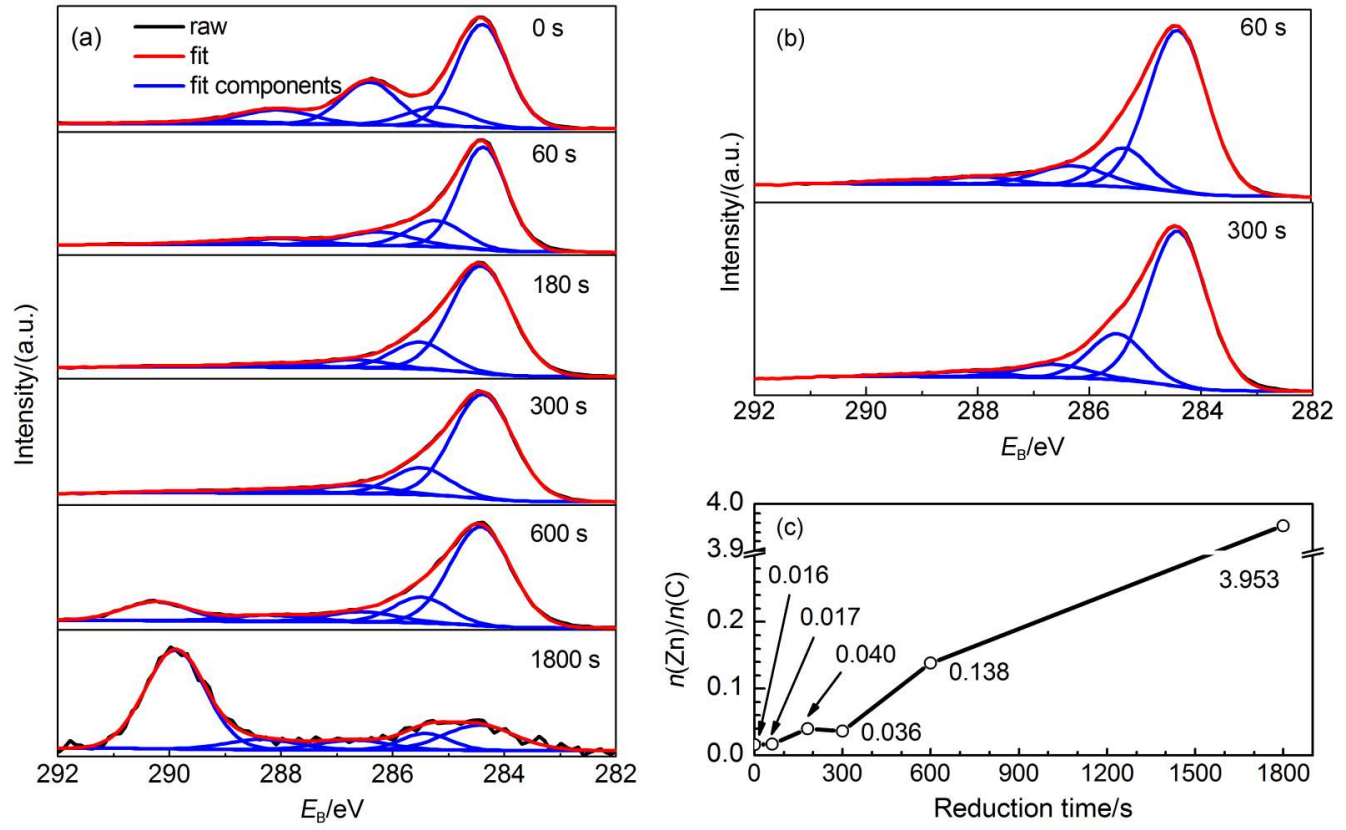

图 2 (a) 不同还原时间 $(0,60,180,300,600,1800 \mathrm{~s})$ 复合膜表面的 C $1 \mathrm{~s}$ XPS 图谱; (b) 60 和 $300 \mathrm{~s}$ 复合膜体相的

$\mathrm{C} 1 \mathrm{~s}$ XPS 图谱; (c) 复合膜表面 $\mathrm{ZnO}$ 与 $\mathrm{GO}$ 摩尔比 $(\mathrm{Zn}$ 与 $\mathrm{C}$ 原子比)随还原时间的变化曲线

Fig.2 (a) C $1 s$ XPS spectra of the surfaces of the composite films with different reduction time $(0,60,180,300,600,1800$ s); (b) C $1 s$ XPS spectra of the 60 and 300 s composites after etching; (c) the variation curve of the molar ratio of

$\mathrm{ZnO}$ to GO (atomic ratio of $\mathrm{Zn}$ to $\mathrm{C}$ ) of the film surfaces with reduction time

The fit components are $\mathrm{C} s p^{2}, \mathrm{C} s p^{3} \&$ hydroxyl $\mathrm{C}-\mathrm{OH}$, epoxy $\mathrm{C}-\mathrm{O}-\mathrm{C}$, carbonyl $\mathrm{C}=\mathrm{O}$, and carboxyl $\mathrm{COOH}$ with increasing order of the binding energy.

表 1 GO-ZnO 复合膜表面的 C $1 s$ XPS 拟合峰结合能及其含量

Table 1 Summary of the binding energies $\left(E_{\mathrm{B}}\right)$ and percentages of the components fitted in the $\mathrm{C} 1 s$ XPS spectra of the surfaces of the GO-ZnO composite films

\begin{tabular}{|c|c|c|c|c|c|c|c|c|c|c|c|c|}
\hline & \multicolumn{2}{|r|}{$0 \mathrm{~s}$} & \multicolumn{2}{|r|}{$60 \mathrm{~s}^{*}$} & \multicolumn{2}{|r|}{$180 \mathrm{~s}$} & \multicolumn{2}{|r|}{$300 \mathrm{~s}^{*}$} & \multicolumn{2}{|r|}{$600 \mathrm{~s}$} & \multicolumn{2}{|r|}{$1800 \mathrm{~s}$} \\
\hline & $E_{\mathrm{B}} / \mathrm{eV}$ & Percentage/\% & $E_{\mathrm{B}} / \mathrm{eV}$ & Percentage $/ \%$ & $E_{\mathrm{B}} / \mathrm{eV}$ & Percentage/\% & $E_{\mathrm{B}} / \mathrm{eV}$ & Percentage $/ \%$ & $E_{\mathrm{B}} / \mathrm{eV}$ & Percentage $/ \%$ & $E_{\mathrm{B}} / \mathrm{eV}$ & Percentage $/ \%$ \\
\hline \multirow[t]{2}{*}{$\mathrm{C} s p^{2}$} & 284.4 & 52.00 & 284.4 & 62.20 & 284.4 & 73.13 & 284.4 & 71.41 & 284.4 & 60.37 & 284.4 & 18.04 \\
\hline & & & 284.4 & 70.28 & & & 284.4 & 69.50 & & & & \\
\hline \multirow[t]{2}{*}{$\mathrm{C} s p^{3} \& \mathrm{C}-\mathrm{OH}$} & 285.2 & 11.51 & 285.2 & 17.98 & 285.5 & 16.64 & 285.5 & 17.41 & 285.5 & 14.42 & 285.4 & 8.21 \\
\hline & & & 285.4 & 14.09 & & & 285.5 & 19.60 & & & & \\
\hline \multirow[t]{2}{*}{$\mathrm{C}-\mathrm{O}-\mathrm{C}$} & 286.4 & 23.62 & 286.3 & 11.94 & 286.6 & 6.22 & 286.7 & 6.41 & 286.6 & 7.24 & 286.7 & 6.90 \\
\hline & & & 286.3 & 10.01 & & & 286.7 & 7.29 & & & & \\
\hline \multirow[t]{2}{*}{$\mathrm{C}=\mathrm{O}$} & 288.1 & 9.91 & 288.0 & 5.53 & 288.2 & 2.86 & 288.2 & 3.16 & 288.2 & 5.20 & 288.3 & 7.35 \\
\hline & & & 288.0 & 3.88 & & & 288.2 & 2.99 & & & & \\
\hline \multirow[t]{2}{*}{$\mathrm{COOH}$} & 289.2 & 2.95 & 289.3 & 2.35 & 289.8 & 1.14 & 289.8 & 1.61 & 290.2 & 12.76 & 289.9 & 59.49 \\
\hline & & & 289.4 & 1.74 & & & 289.5 & 0.61 & & & & \\
\hline
\end{tabular}

"The first and second lines denote the information of the GO-ZnO film before and after etching, respectively.

180 s GO 氧官能团含量继续下降, 还原程度进一 步增加。300 s GO 氧官能团相对含量与 $180 \mathrm{~s} \mathrm{GO}$ 基本一致, 还原程度未发生改变, 暗示了该还原 程度为 $-2.1 \mathrm{~V}$ 电位条件下的极限。随时间继续增 加, $600 \mathrm{~s} \mathrm{GO}$ 中的羧基出现增多, GO 的还原程度 反而减小。1 $1800 \mathrm{~s} \mathrm{GO}$ 中的羧基百分含量急剧增 加, 并远超 $s p^{2}-\mathrm{C}$ 的含量。由对复合膜 GO 的 FTIR 表征(图 S4)结果可见, 从 $300 \mathrm{~s}$ 开始, 位于 1714 $\mathrm{cm}^{-1}$ 的 $\mathrm{C}=\mathrm{O}$ 伸缩振动峰 ${ }^{22}$ 在增大的同时, $670 \mathrm{~cm}^{-1}$ 处芳香环 $\mathrm{C}-\mathrm{H}$ 振动峰 ${ }^{22}$ 的相对强度也不断增加。 由于以上两化学键均位于 $\mathrm{GO}$ 边缘, 它们的同时增 加暗示了 $\mathrm{GO}$ 边缘的增多, 即 $\mathrm{GO}$ 片层可能出现了 断裂和破碎。

为更加精细地考察 $\mathrm{GO}$ 结构的演变, 对 $60 \mathrm{~s}$ 和 $300 \mathrm{~s}$ 样品进行深度剖析, 得到溅射后(刻蚀深度约 $50 \mathrm{~nm}$ )的 C $1 s$ XPS 图谱如图 2(b)所示, 相应的拟合 
信息仍列于表 1 。对 $60 \mathrm{~s} \mathrm{GO}$, 其体相氧官能团相 对含量较表面略微下降, 这与文献 ${ }^{16}$ 报道中 $\mathrm{GO}$ 的 电化学还原优先从祄底附近开始的结论一致。然 而该 $\mathrm{GO}$ 中还原程度的梯度随厚度变化较小, 且体 相 GO 的还原程度仍远小于 $180 \mathrm{~s} \mathrm{GO}$ 的最大还原程 度, 这可能与 $\mathrm{GO}$ 膜厚度较小有关。由此我们近似 认为电化学还原是对整个 $\mathrm{GO}$ 膜均匀进行的。对 $300 \mathrm{~s} \mathrm{GO}$, 其体相还原程度与表面基本一致, 证明 此时整个 GO 膜均处于最大还原态, 并未发生进一 步结构转变。根据以上结果, 我们断定随电化学 处理时间增加, 整个 $\mathrm{GO}$ 薄膜结构与表面 $\mathrm{GO}$ 结构 的变化趋势一致, 即 $\mathrm{GO}$ 膜经还原达到最大还原态 后, 还原程度保持一段时间, 之后出现下降。前 已述及电化学还原过程的电流密度曲线与文献报 道类似。已知文献 ${ }^{17}$ 所述电流密度的变化仅与 $\mathrm{GO}$ 的还原过程有关, 当达到最大还原程度后, GO 结 构不再变化, 对应的电流密度也不再改变。然 而, 本文电流下降的部分曲线对应 $\mathrm{GO}$ 的化学结构 不变; $600 \mathrm{~s}$ 后电流密度基本稳定, 对应 $\mathrm{GO}$ 的化 学结构却不断变化。由此可见, 两曲线走势相似 的现象仅为巧合。鉴于文献 ${ }^{10,12,17}$ 中的 GO 以导体为 祄底, 我们推测本文 $\mathrm{GO}$ 的变化可能与 $\mathrm{ZnO}$ 半导体 作为祄底有关。鉴于 $Z n 2 p$ 化学状态在处理过程中 不变的现象(图 $\mathrm{S} 5$ ), 我们推测 $\mathrm{ZnO}$ 未参与 $\mathrm{GO}$ 的反
应, 故而 $\mathrm{GO}$ 的羧基化可能包含电解质 $\mathrm{LiClO}_{4}$ 中氧 元素的贡献。关于 $\mathrm{GO}$ 在 $\mathrm{ZnO}$ 祄底上的结构随还原 时间的变化机理仍在实验探讨中。

根据表面 XPS 数据, 得到表面 $\mathrm{ZnO}$ 与 $\mathrm{GO}$ 摩尔 比 $(\mathrm{Zn}$ 与 $\mathrm{C}$ 原子比, 下同)随还原时间的变化曲线如 图 2(c)所示。对未还原的 $\mathrm{GO}-\mathrm{ZnO}$ 复合膜, $\mathrm{Zn}$ 与 $\mathrm{C}$ 原子比仅为 0.016 , 这与 $\mathrm{GO}$ 膜覆盖在 $\mathrm{ZnO}$ 层上方 造成对 $\mathrm{ZnO}$ 组分的掩盖有关。随还原进行, 60、 180 和 $300 \mathrm{~s}$ 样品的 $\mathrm{Zn}$ 与 $\mathrm{C}$ 原子比依次为 0.017 、 0.040 和 0.036 , 仅出现略微增加; 600 和 $1800 \mathrm{~s}$ 样 品的 $\mathrm{Zn}$ 与 $\mathrm{C}$ 原子比急剧增大, 分别为 0.138 和 3.953。后两组样品 $\mathrm{Zn}$ 与 $\mathrm{C}$ 原子比的剧烈增加暗示 了 $\mathrm{GO}$ 膜对 $\mathrm{ZnO}$ 掩盖作用的减弱, 这可能正是由 $\mathrm{GO}$ 出现部分碎裂, 小尺寸的 $\mathrm{GO}$ 填入到 $\mathrm{ZnO}$ 纳米 柱阵列的间隙, 从而使更多的 $\mathrm{ZnO}$ 暴露在表面造 成的。

图 3 给出了各 GO-ZnO 复合膜的 FESEM 表面 形貌。与 $0 \mathrm{~s}$ 样品相比, $60 \mathrm{~s}$ 样品形貌基本不变, 在 $\mathrm{GO}$ 叠层上可见少量小颗粒; 随后的 180 和 $300 \mathrm{~s}$ 样品 $\mathrm{GO}$ 叠层上的颗粒逐渐增大和增多, 至 $600 \mathrm{~s}$ 样品表面基本完全被颗粒覆盖; $1800 \mathrm{~s}$ 样品表面可 见 $\mathrm{GO}$ 叠层形貌的消失, $\mathrm{GO}$ 片层不再连续并出现 部分团聚(圈中区域), 纳米柱轮廓也隐约可见。由 前文分析, 60 与 $0 \mathrm{~s}$ 样品形貌相似的现象与 $\mathrm{GO}$ 仅

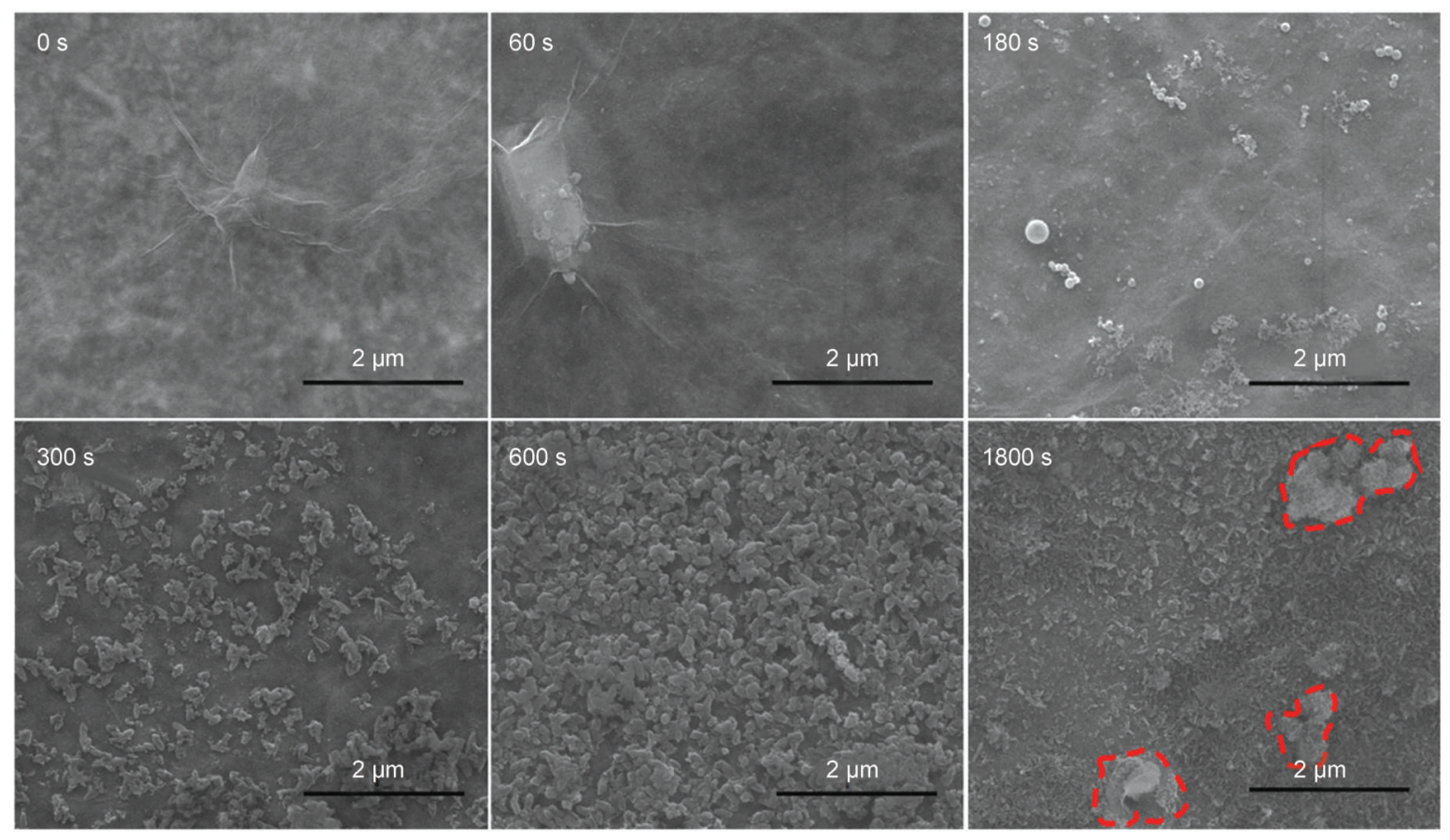

图 3 不同还原时间的 GO-ZnO复合膜表面形貌的 FESEM 图

Fig.3 FESEM images of the GO-ZnO composite film surfaces with different reduction time 
产生化学结构的改变有关。对 180 和 $300 \mathrm{~s}$ 样品, 前文指出其化学结构不变。然而, 由于还原后的 $\mathrm{GO}$ 具有电荷储存性能 ${ }^{23}$, 其对电子的存储随时间 增加而不断增加, 从而可能使 $\mathrm{GO}$ 褶皱程度不断增 大并产生大的团聚颗粒。另外, GO 存储电荷量的 逐渐饱和将导致电流密度的不断减小。仍根据前 文分析, $600 \mathrm{~s} \mathrm{GO}$ 中可能已经出现了部分片层破 碎的现象。然而, 其团聚颗粒仍沿前几组样品走 势变化的现象表明, 破碎 $\mathrm{GO}$ 的浓度较为有限, 即 $\mathrm{GO}$ 平均尺寸并未显著减小。1 $1800 \mathrm{~s}$ 样品的表面形 貌与前文 $\mathrm{GO}$ 片层大量破碎并生成小尺寸 $\mathrm{GO}$ 的结 论对应, 其中的团聚可能是由 $\mathrm{GO}$ 边缘羧基急剧增 多，使 GO片层间的氢键作用增强造成的。

对各复合膜的光学性质进行比较, 得到 UVVis 透过率曲线如图 4(a)所示。由图可见, $0 \mathrm{~s}$ 样品 的可见透过率最大, 60 到 $300 \mathrm{~s}$ 样品的透过率依次 减小, $600 \mathrm{~s}$ 样品的透过率出现微弱增加, 而 1800 $\mathrm{s}$ 样品的透过率又减小。由于各样品祄底的吸收性 质一致, 且 $\mathrm{GO}$ 膜厚度相同, 以上透过率差异应主 要与 $\mathrm{GO}$ 化学结构和微观形貌的改变有关。从图 4 (b)各复合膜的 Tauc 曲线可见, GO 的间接光学带 隙 ${ }^{24}$ 随时间增加依次为 $3.08 \mathrm{eV}(0 \mathrm{~s}), 2.30 \mathrm{eV}(60$ s), $2.13 \mathrm{eV}(180 \mathrm{~s}), \quad 1.87 \mathrm{eV}(300 \mathrm{~s}), 2.40 \mathrm{eV}(600$ $\mathrm{s})$ 和 $1.90 \mathrm{eV}(1800 \mathrm{~s})$ 。对 $0 、 60$ 和 $180 \mathrm{~s} \mathrm{GO,}$, 随还
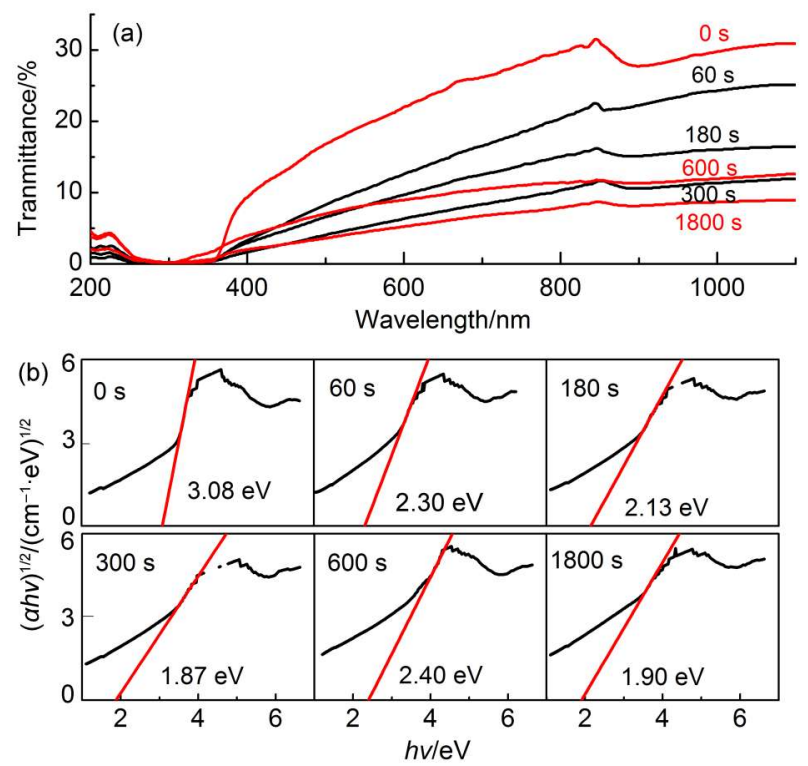

图 4 (a)不同复合膜样品的 UV-Vis 透过率曲线; (b)不同复合膜样品的 Tauc曲线

Fig.4 (a) UV-Vis transmittance spectra of different composite films; (b) Tauc plots of different composite films
原程度逐渐增加, 光学带隙不断减小, 使光吸收 不断增加, 并与透过率曲线下降的趋势对应。对 $300 \mathrm{~s} \mathrm{GO}$, 其还原程度与 $180 \mathrm{~s}$ 相同而带隙继续减 小。我们推测两者带隙的差异可能源自 GO 膜微观 形貌的改变。实际上, 文献 ${ }^{25}$ 曾提到形变可引起石 墨烯片层应力的改变, 并使基面晶格常数产生变 化, 从而造成能隙的改变。对 $600 \mathrm{~s} \mathrm{GO}$, 虽然其 微观形貌进一步变化, 但其片层边缘羧基基团的 增多使还原程度减小可能是造成带隙增大及光吸 收减小的主因。已知 $600 \mathrm{~s} \mathrm{GO}$ 的带隙大于 $60 \mathrm{~s}$ GO, 即前者对可见光的吸收应少于后者, 然而前 者透过率较后者大幅减小，表明 $600 \mathrm{~s} \mathrm{GO}$ 对可见 光具有较强的散射, 并可能源自薄膜中的大量团

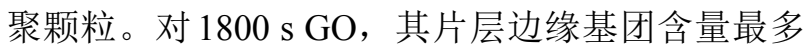
使还原程度最小, 然而其带隙较 $600 \mathrm{~s} \mathrm{GO}$ 出现减 小。此现象可能由 $\mathrm{GO}$ 尺寸的大幅减小引起, 并暗 示了其能级结构与前几组样品可能存在较大差异。

对各样品进行电化学测试(详见图 S6), 分别得 到 $\mathrm{GO}$ 的导带底(CBM), 价带顶(VBM) 和费米能 级, 并作其能级结构随时间变化的示意图如图 5 所 示。图中，位于 $\mathrm{H} / \mathrm{H}^{+}$电化学势(虚线)上方的实线表 示 $\mathrm{CBM}$ ，位于其下方的实线表示 VBM，位于两者 之间的点线则表示费米能级。对各 GO 的能级结 构, 首先由 $\mathrm{CBM}$ 与 $\mathrm{VBM}$ 的差值得到还原后 $\mathrm{GO}$ 的 能隙(如图 5 所示 $E_{\mathrm{g}}$ 值)与光学带隙基本一致。其 次, 根据费米能级的位置可得, $0 \mathrm{~s} \mathrm{GO}$ 的载流子 极性为 $p$ 型, 60 到 $600 \mathrm{~s} \mathrm{GO}$ 均转变为 $n$ 型, 至 $1800 \mathrm{~s} \mathrm{GO}$ 又回到 $p$ 型。已知 $\mathrm{GO}$ 的半导极性由 GO 结构中各类氧官能团的相对含量决定 ${ }^{26}$ 。由前文 XPS 分析, $60 \mathrm{~s}$ 到 $600 \mathrm{~s} \mathrm{GO}$ 的 $n$ 型导电性质与吸电

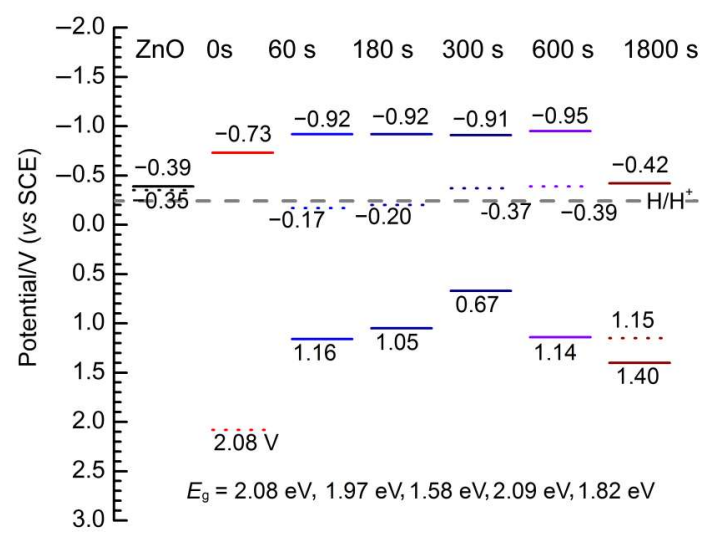

图 $5 \mathrm{ZnO}$ 及不同还原时间 GO 的能级结构示意图

Fig.5 Energy level structures of $\mathrm{ZnO}$ and $\mathrm{GO}$ with different reduction time 
子的环氧基、羰基及羧基等基团含量的大幅降低 有关, 而 $1800 \mathrm{~s} \mathrm{GO}$ 中大量吸电子羧基基团的生成 可能是其重回 $p$ 型导电性质的主因。将 $\mathrm{GO}$ 能级与 $\mathrm{ZnO}$ 能级进行比较可见, 除 $1800 \mathrm{~s} \mathrm{GO}$ 外, 其他样 品 $\mathrm{GO}$ 的 $\mathrm{CBM}$ 均高于 $\mathrm{ZnO}$, 显示了 $\mathrm{GO}$ 平均尺寸的 变化对能级结构的影响。另外, 0、60、180 和 $1800 \mathrm{~s} \mathrm{GO}$ 的费米能级均低于 $\mathrm{ZnO}$, 故而在 $\mathrm{GO}$ 和 $\mathrm{ZnO}$ 界面可产生由 $\mathrm{ZnO}$ 指向 $\mathrm{GO}$ 的正向内建电场。 300 和 $600 \mathrm{~s} \mathrm{GO}$ 的费米能级略高于 $\mathrm{ZnO}$, 两者接触 后则会在界面产生微弱的反向电场。通过以上对 $\mathrm{GO}$ 的能级分析可得, 当以光子激发 $\mathrm{GO}$ 时, 各 $\mathrm{GO}-\mathrm{ZnO}$ 复合膜将表现出不同的光电转换行为。

以复合膜为工作电极, $\mathrm{Pt}$ 丝和 $\mathrm{Ag} / \mathrm{Ag}^{+}$电极分 别为对电极和参比电极组装光电化学池进行光电 流测试。当电解液中仅含支持电解质时, 测试体 系的光电响应可直接反映光电极的性质 ${ }^{23}$ 。采用 $0.1 \mathrm{~mol} \cdot \mathrm{L}^{-1} \mathrm{LiClO}_{4}$ 的乙腈溶液作为电解液, 得到各 样品电流密度的响应曲线如图 6(a)所示, 电流密度 的平均值列于表 2 中。其中, $\mathrm{ZnO}$ 膜上出现的微弱 电流可能与其杂质能级的激发有关 ${ }^{27}$ 。在复合膜 中, 由于祄底 $\mathrm{ZnO}$ 的结构相同, 各样品光电响应 的差异只与 $\mathrm{GO}$ 有关。从电流方向来看, 它们的差 异取决于 GO 的能级结构。0、60和 $180 \mathrm{~s}$ 样品产生 阳极电流(方向由电极指向溶液), 主要源于 GO$\mathrm{ZnO}$ 界面产生的正向内建电场的驱动及相对较高 的 GO 导带对电子跃迁的零势垒(见图 5)。300、 600 和 $1800 \mathrm{~s}$ 样品出现阴极电流, 其中前两组电流 的反向源于 $\mathrm{GO}-\mathrm{ZnO}$ 界面反向电场的作用; $1800 \mathrm{~s}$ 样品中, 尽管 $\mathrm{GO}-\mathrm{ZnO}$ 界面存在正向电场, 但 $\mathrm{GO}$ 导带位置相对较低, 从而使 $\mathrm{GO}$ 上电子向 $\mathrm{ZnO}$ 的迁 移被界面势垒阻挡, 向溶液泄露的少量反向电子 则形成电流(漏电流)。

从电流密度来看, 它们的差异与 $\mathrm{GO}$ 的吸光和 导电以及 $\mathrm{GO}$ 与 $\mathrm{ZnO}$ 的接触状态有关。由表 2 可 见, 各复合膜样品的光电流密度绝对值均小于 1 $\mu \mathrm{A} \cdot \mathrm{cm}^{-2}$ 。根据我们之前的研究, $\mathrm{GO}$ 膜极差的层 间导电性和与祄底较小的接触面积是造成光生电 荷分离效率降低, 并导致光电流密度减小的主 因 ${ }^{13}$ 。在前三组阳极电流中, $60 \mathrm{~s}$ 样品的电流密度 最大。对 $0 \mathrm{~s}$ 样品, 由 $\mathrm{GO}$ 的能隙 $(3.08 \mathrm{eV})$ 计算得其 吸收截止波长约为 $400 \mathrm{~nm}$, 即在本文光照条件下 不能产生本征激发。因此, 样品的光响应仍主要 源自 $\mathrm{ZnO}$ 的微弱激发。其电流密度 $(-0.067 \mu \mathrm{A}$.
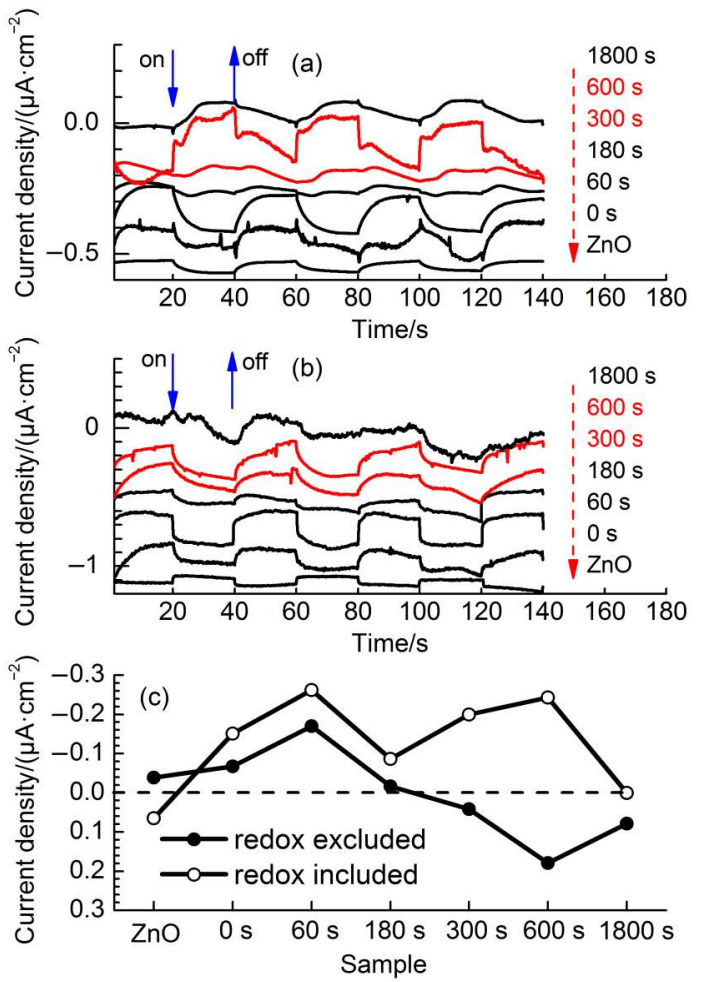

图 6 各薄膜在不包含 (a)和包含 (b)氧化-还原电对的电解液 中的可见光电流密度-时间曲线; (c) 薄膜光电流密度随 样品的变化曲线在两种测试溶液中的对比

Fig.6 Transient photocurrent density-time curves of the films measured in the electrolyte without (a) and with (b) redox couples; (c) comparison of the current density variation curves of samples measured in the electrolyte without and with redox couples

$\left.\mathrm{cm}^{-2}\right)$ 较 $\mathrm{ZnO}$ 膜 $\left(-0.039 \mu \mathrm{A} \cdot \mathrm{cm}^{-2}\right)$ 的增加可能是 $\mathrm{ZnO}$ 上的光生空穴向 $\mathrm{GO}$ 转移, 使空穴与电子的复合有 所减弱的缘故。60和 $180 \mathrm{~s}$ 样品的 GO 均可吸收可 见光, 且后者的吸收大于前者(见图 4(a)); 然而前 者电流密度 $\left(-0.170 \mu \mathrm{A} \cdot \mathrm{cm}^{-2}\right)$ 远大于后者 $(-0.016$ $\left.\mu \mathrm{A} \cdot \mathrm{cm}^{-2}\right)$ 。造成以上现象的原因可能是后者 $\mathrm{GO}$ 达 到最大还原程度后, $\mathrm{GO}$ 膜中各叠层间氧官能团的 减少造成层间结合力减弱, 使层间导电性更差, 从而可能造成光生电荷在传输时的大量湮灭。在 后三组阴极电流中, $600 \mathrm{~s}$ 样品的电流密度最大 $\left(0.179 \mu \mathrm{A} \cdot \mathrm{cm}^{-2}\right)$ 。经过对比可见, 300 和 $600 \mathrm{~s}$ 样品 与前两组 $(60$ 和 $180 \mathrm{~s})$ 样品的情况类似, 即前者光 吸收大于后者(仍见图 4(a)) 而电流密度却相反(见表 2)。与 $300 \mathrm{~s} \mathrm{GO}$ 膜相比, $600 \mathrm{~s} \mathrm{GO}$ 膜的部分破碎可 能使叠层形貌受到一定程度的破坏, 从而使部分 光生电荷在膜内的传导可沿着导电性更好的 GO 片 层内进行; 一些 $\mathrm{GO}$ 碎片还可填入到祄底空隙, 从 而增加了 $\mathrm{GO}$ 膜与 $\mathrm{ZnO}$ 的接触面积, 并使电荷在 
表 2 各薄膜在不含以及包含电对的电解液中的平均光电流密度

Table 2 Average photocurrent density of different films measured in electrolytes without and with redox couples

\begin{tabular}{cccccccc}
\hline & \multicolumn{5}{c}{ Average photocurrent density $/\left(\mu \mathrm{A} \cdot \mathrm{cm}^{-2}\right)$} \\
\cline { 2 - 6 } & $\mathrm{ZnO}$ & $0 \mathrm{~s}$ & $60 \mathrm{~s}$ & $180 \mathrm{~s}$ & $300 \mathrm{~s}$ & $600 \mathrm{~s}$ \\
\hline redox excluded & -0.039 & -0.067 & -0.170 & -0.016 & 0.042 & 0.179 & 0.079 \\
redox included & 0.065 & -0.151 & -0.262 & -0.086 & -0.200 & -0.243 & 0 \\
\hline
\end{tabular}

GO-ZnO 界面的迁移更加顺畅。由此, $600 \mathrm{~s}$ 样品 中的光生载流子可更快地产生界面分离, 并使复 合几率降低可能是其电流密度较大的原因。实际 上, 随光照开关, $600 \mathrm{~s}$ 样品电流随光照开关的响 应速度最快的现象(图 6(a)) 也佐证了以上推测。 $1800 \mathrm{~s}$ 样品光电流密度的贡献来源于漏电流, 故数 值较小。

向上述电解液加入氧化还原电对 $\left(10 \mathrm{mmol} \cdot \mathrm{L}^{-1}\right.$ LiI 和 $\left.1 \mathrm{mmol} \cdot \mathrm{L}^{-1} \mathrm{I}_{2}\right)$, 得到各样品的电流密度响应 曲线如图 6(b)所示, 电流密度的平均值仍列于表 2。与前一组电解液相比, $\mathrm{ZnO}$ 膜电流反向和 $1800 \mathrm{~s}$ 复合膜电流为 0 的原因未知。其余各复合膜 的光电流均为阳极电流, 这可能与碘离子电对的 电化学势有关一一当电对的电化学势(约 $0.16 \mathrm{~V}$ ( $\mathrm{vs}$ $\mathrm{SCE})^{28}$ ) 低于 $\mathrm{GO}$ 的费米能级时 $(60$ 至 $600 \mathrm{~s})$, 测试体 系的电场可由原来 $\mathrm{GO}-\mathrm{ZnO}$ 界面的内建电场转变为 $\mathrm{ZnO}$ 费米能级与该电化学势之差, 且方向指向正 向。将两组电解液中的电流密度随样品的变化曲 线进行对比(图 6(c)), 可见电流密度绝对值的走势 相同。其中, 电流密度值在含电对的溶液中较大 (如表 2 所示), 这可能与碘离子电对可有效转移溶 液界面积累的光生空穴, 使其与光生电子复合的 几率降低有关。

由光电化学测试结果可见, 在以 $\mathrm{ZnO}$ 为电子 导体的复合膜中, 虽然仅有 60 和 $180 \mathrm{~s} \mathrm{GO}$ 可作为 空穴导体与 $\mathrm{ZnO}$ 复合组装双结电池, 60 至 $600 \mathrm{~s}$ $\mathrm{GO}$ 则均可作为吸光体与 $\mathrm{ZnO}$ 及第三方空穴导体 (此处为碘离子电对)共同复合组装三结电池, 表明 $\mathrm{GO}$ 在太阳光电转换领域的广泛适用性。实际上, $1800 \mathrm{~s}$ 样品中 $\mathrm{GO}$ 叠层完全破坏, 造成 $\mathrm{GO}$ 中的电 荷传导以片层内传导为主; 小尺寸的 $\mathrm{GO}$ 大量填入 祄底空隙, 造成界面面积剧烈增加。以上优势应 使其载流子分离效率最高。因此, 在 $\mathrm{ZnO}$ 祄底 上, 利用 $\mathrm{GO}$ 在电化学还原时可产生破碎的现象, 制备同时具有导带高于 $\mathrm{ZnO}$ 和无序填隙形貌的 $\mathrm{GO}$ 吸光体是获得具有良好光电转换性能的阳极光电 极的关键。

\section{4 结 论}

考察了在有机溶液中, $\mathrm{ZnO}$ 祄底上的 $\mathrm{GO}$ 膜结 构随电化学还原时间的演变过程, 以及不同 GO$\mathrm{ZnO}$ 复合膜的光电转换性能, 经讨论后得到结论 如下：(1) 在电化学处理过程中, $\mathrm{ZnO}$ 半导体上 $\mathrm{GO}$ 经还原到最大程度后, 会产生进一步物理状态的 改变; 随反应时间的继续延长产生碎裂, 生成小 尺寸 GO, 并在边缘生成大量双键氧官能团; (2) $\mathrm{GO}$ 化学结构和物理禇皱的变化均会造成 GO 能级 结构的改变; (3) 对不同的复合膜光电极, $\mathrm{GO}$ 导 带和费米能级相对于 $\mathrm{ZnO}$ 的位置共同决定了电极 的电流方向; (4) GO 的碎片化可造成 GO 片层的无 序分布, 并促进 $\mathrm{GO}$ 向 $\mathrm{ZnO}$ 空隙的填充以增加 $\mathrm{GO}$ 与 $\mathrm{ZnO}$ 的接触, 从而有利于 $\mathrm{GO}$ 上光生电荷向 $\mathrm{ZnO}$ 的转移, 并提高光电转换性能。与当前导体 (金 属、导电塑料等)或简并半导体上(FTO 和 ITO等)的 $\mathrm{GO}$ 在电化学条件下仅产生还原的现象相比, $\mathrm{ZnO}$ 半导体上的 $\mathrm{GO}$ 在还原过程中反常的结构演变为电 化学调控 GO 结构的研究提供了新的手段, 其中 $\mathrm{GO}$ 的破碎现象除可被用来制备不同尺寸和能级结 构的 GO 外, 也为氧化石墨烯量子点的制备和 GO 的羧基化研究提供了新的思路。

Supporting Information: available free of charge via the internet at http://www.whxb.pku.edu.cn

\section{References}

(1) Dreyer, D. R.; Park, S.; Bielawski, C. W.; Ruoff, R. S. Chem. Soc. Rev. 2010, 39, 228. doi: 10.1039/b917103g

(2) Huang, H.; Li, Z.; She, J.; Wang, W. J. Appl. Phys. 2012, 111, 054317. doi: 10.1063/1.3694665

(3) Lahaye, R. J. W. E.; Jeong, H. K.; Park, C. Y.; Lee, Y. H. Phys. Rev. B 2009, 79, 125435 doi: 10.1103/PhysRevB.79.125435

(4) Bagri, A.; Mattevi, C.; Acik, M.; Chabal, Y. J.; Chhowalla, M.; Shenoy, V. B. Nat. Chem. 2010, 2, 581. doi: 10.1038/ NCHEM.686

(5) Loh, K. P.; Bao, Q.; Eda, G.; Chhowalla, M. Nat. Chem. 2010, 2, 1015. doi: 10.1038/nchem.907

(6) Shen, Y.; Yang, S.; Zhou, P.; Sun, Q.; Wang, P.; Wan, L.; Li, J.; 
Chen, L.; Wang, X.; Ding, S.; Zhang, D. W. Carbon 2013, 62, 157. doi: 10.1016/j.carbon.2013.06.007

(7) Chang, H.; Sun, Z.; Saito, M.; Yuan, Q.; Zhang, H.; Li, J.; Wang, Z.; Fujita, T.; Ding, F.; Zheng, Z.; Yan, F.; Wu, H.; Chen, M.; Ikuhara, Y. ACS Nano 2013, 7 (7), 6310. doi: 10.1021/ nn4023679

(8) Yeh, T. F.; Syu, J. M.; Cheng, C.; Chang, T. H.; Teng, H. Adv. Funct. Mater. 2010, 20, 2255. doi: 10.1002/adfm.201000274

(9) Hsu, H. C.; Shown, I.; Wei, H. Y.; Chang, Y. C.; Du, H. Y.; Lin, Y. G.; Tseng, C. A.; Wang, C. H.; Chen, L. C.; Lin, Y. C.; Chen, K. H. Nanoscale 2013, 5, 262. doi: 10.1039/c2nr31718d

(10) Pei, S.; Cheng, H. M. Carbon 2012, 50, 3210. doi: 10.1016/j. carbon.2011.11.010

(11) Dreyer, D. R.; Murali, S.; Zhu, Y.; Ruoff, R. S.; Bielawski, C. W. J. Mater. Chem. 2011, 21, 3443. doi: 10.1039/c0jm02704a

(12) Kauppila, J.; Kunnas, P.; Damlin, P.; Viinikanoja, A.; Kvarnström, C. Electrochim. Acta 2013, 89, 84. doi: 10.1016/j. electacta.2012.10.153

(13) Li, W. Y.; He, Y. Q.; Li, Y. M. Acta Phys. -Chim. Sin. 2015, 31 (3), 457. [李文有, 贺蕴秋, 李一鸣. 物理化学学报, 2015, 31 (3), 457.] doi: 10.3866/PKU.WHXB201501093

(14) Chen, C.; Cai, W.; Long, M.; Zhou, B.; Wu, Y.; Wu, D.; Feng, Y. ACS Nano 2010, 4 (11), 6425. doi: 10.1021/nn102130m

(15) Li, Y.; Wang, D.; Li, W.; He, Y. J. Alloy. Compd. 2015, 648, 942. doi: 10.1016/j.jallcom.2015.07.037

(16) Wang, X.; Kholmanov, I.; Chou, H.; Ruoff, R. S. ACS Nano 2015, 9 (9), 8737. doi: 10.1021/acsnano.5b03814

(17) Harima, Y.; Setodoi, S.; Imae, I.; Komaguchi, K.; Ooyama, Y.; Ohshita, J.; Mizota, H.; Yano, J. Electrochim. Acta 2011, 56,
5363. doi: j.electacta.2011.03.117

(18) Zhou, M.; Wang, Y.; Zhai, Y.; Zhai, J.; Ren, W.; Wang, F.; Dong, S. Chem. Euro. J. 2009, 15 (25), 6116. doi: 10.1002/ chem.200900596

(19) Pradhan, D.; Leung, K. T. Langmuir 2008, 24, 9707. doi: $10.1021 / 1 a 8008943$

(20) Pavlishchuk, V. V.; Addison, A. W. Inorg. Chim. Acta 2000, 298, 97. doi: 10.1016/S0020-1693(99)00407-7

(21) Yu, D.; Yang, Y.; Durstock, M.; Baek, J. B.; Dai, L. ACS Nano 2010, 4 (10), 5633. doi: 10.1021/nn101671t

(22) Kumar, N. A.; Choi, H. J.; Shin, Y. R.; Chang, D. W.; Dai, L.; Baek, J. B. ACS Nano 2012, 6 (2), 1715. doi: 10.1021/ nn204688c

(23) Hayashi, H.; Lightcap, I. V.; Tsujimoto, M.; Takano, M.; Umeyama, T.; Kamat, P. V.; Imahori, H. J. Am. Chem. Soc. 2011, 133, 7684. doi: 10.1021/ja201813n

(24) Yeh, T. F.; Chan, F. F.; Hsieh, C. T.; Teng, H. J. Phys. Chem. C 2011, 115, 22587. doi: 10.1021/jp204856c

(25) Neto, A. H. C.; Guinea, F.; Peres, N. M. R.; Novoselov, K. S.; Geim, A. K. Rev. Mod. Phys. 2009, 81, 109. doi: 10.1103/ RevModPhys.81.109

(26) Tu, N. D. K.; Choi, J.; Park, C. R.; Kim, H. Chem. Mater. 2015, 27, 7362. doi: 10.1021/acs.chemmater.5b02999

(27) Liu, H.; Sun, Q.; Xing, J.; Zheng, Z.; Zhang, Z.; Lü, Z.; Zhao, Z. ACS Appl. Mater. Interfaces 2015, 7, 6645. doi: 10.1021/ am509084r

(28) Yan, X.; Cui, X.; Li, B.; Li, L. Nano Lett. 2010, 10, 1869. doi: $10.1021 / \mathrm{nl} 101060 \mathrm{~h}$ 
Supporting Information for Acta Phys. -Chim. Sin. 2017, 33 (3), 554-562

doi: $10.3866 /$ PKU.WHXB201611171

\title{
氧化石墨烯在氧化锌祄底上的电化学还原及其光电性能
}

\author{
李一鸣 ${ }^{1}$ 陈 肖 ${ }^{1} \quad$ 刘晓军 $^{1} \quad$ 李文有 $^{1} \quad$ 贺蕴秋 ${ }^{1,2,{ }^{*}}$
}

( ${ }^{1}$ 同济大学材料科学与工程学院, 上海 $201804 ;{ }^{2}$ 先进土木工程材料教育部重点实验室, 上海 201804)

\section{Electrochemical Reduction of Graphene Oxide on ZnO Substrate and Its Photoelectric Properties}

\author{
LI Yi-Ming $^{1} \quad$ CHEN Xiao $^{1} \quad$ LIU Xiao-Jun $^{1} \quad$ LI Wen-You $^{1} \quad$ HE Yun-Qiu ${ }^{1,2, *}$ \\ ( ${ }^{1}$ School of Materials Science and Engineering, Tongji University, Shanghai 201804, P. R. China; \\ ${ }^{2}$ Key Laboratory of Advanced Civil Engineering Materials, Ministry of Education, Shanghai 201804, P. R. China)
}

${ }^{*}$ Corresponding author. Email: heyunqiu@ $@$ tongji.edu.cn. 


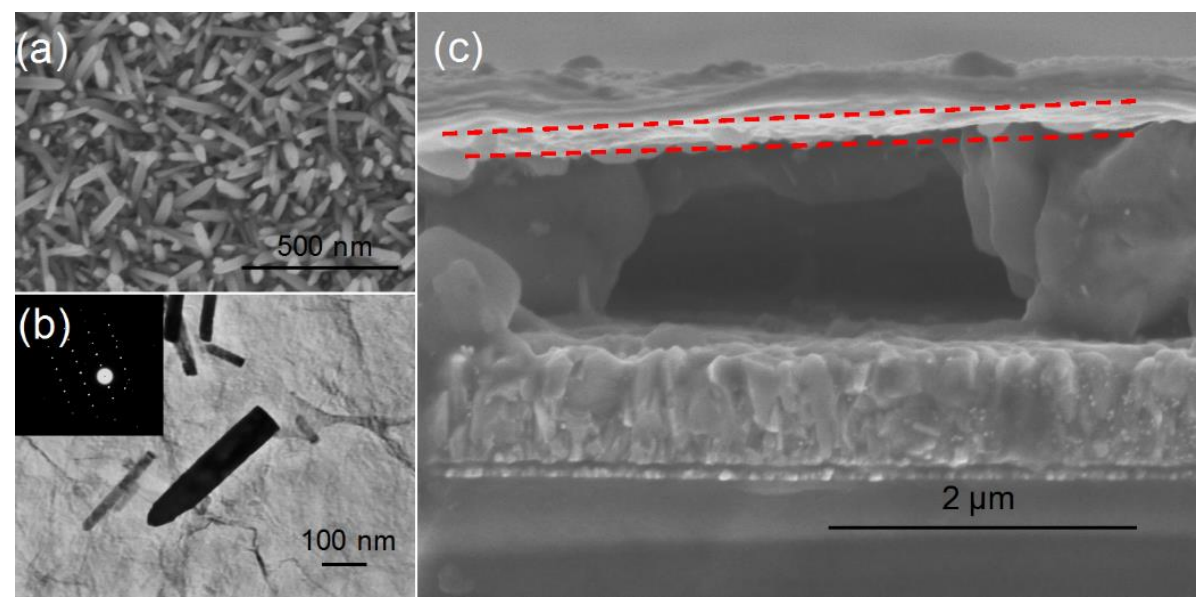

图 S1 (a) ZnO 衬底的表面微观形貌; (b) 从 $\mathrm{GO}-\mathrm{ZnO}$ 复合膜上剥离得到 GO 样 品的 TEM 图像, 图中背景的褶皱为 GO 片层形貌。插图为单个 $\mathrm{ZnO}$ 纳米柱的 选区电子衍射(SAED)斑点; (c) GO 膜的截面形貌

Fig.S1 (a) Micro morphology of the ZnO nanorod array; (b) TEM image of the $\mathrm{ZnO}$ nanorods, with the background image of the wrinkled GO flakes. the inset is the selected area electron diffraction (SAED) image of the single nanorod; (c) The cross-sectional view of the GO layer on the GO-ZnO composite film

(a)

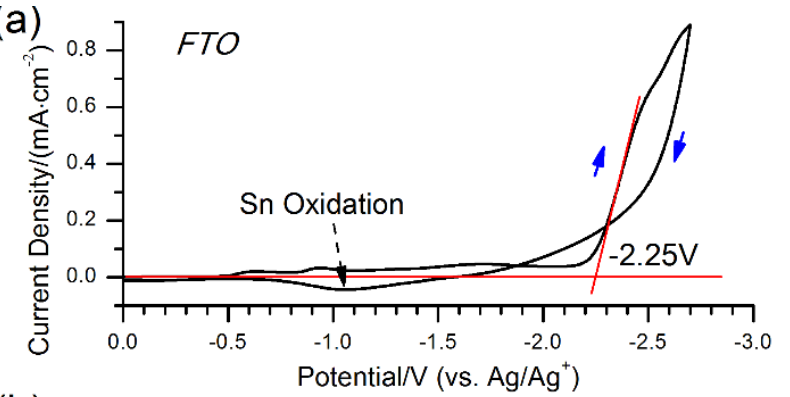

(b)

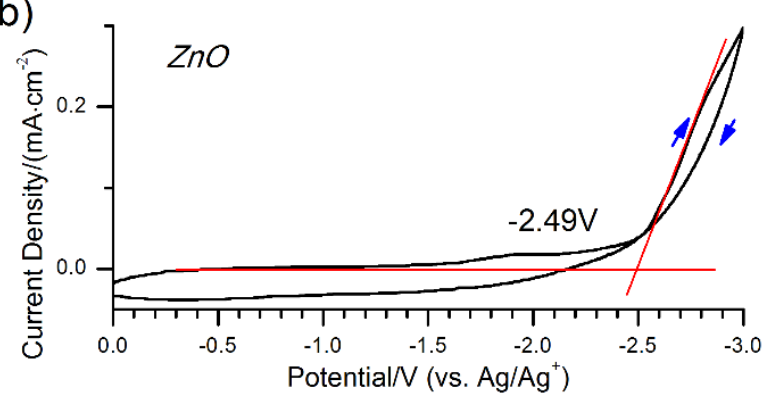

图 S2 (a)和(b)分别为 FTO 玻璃和 ZnO 祄底的循环伏安曲线(扫描速度 10

$$
\left.\mathrm{mV} \cdot \mathrm{s}^{-1}\right)
$$

Fig.S2 (a) and (b) are the cyclic voltammograms of the FTO glass and the $\mathrm{ZnO}$ film with a scan rate of $10 \mathrm{mV} \cdot \mathrm{s}^{-1}$ 


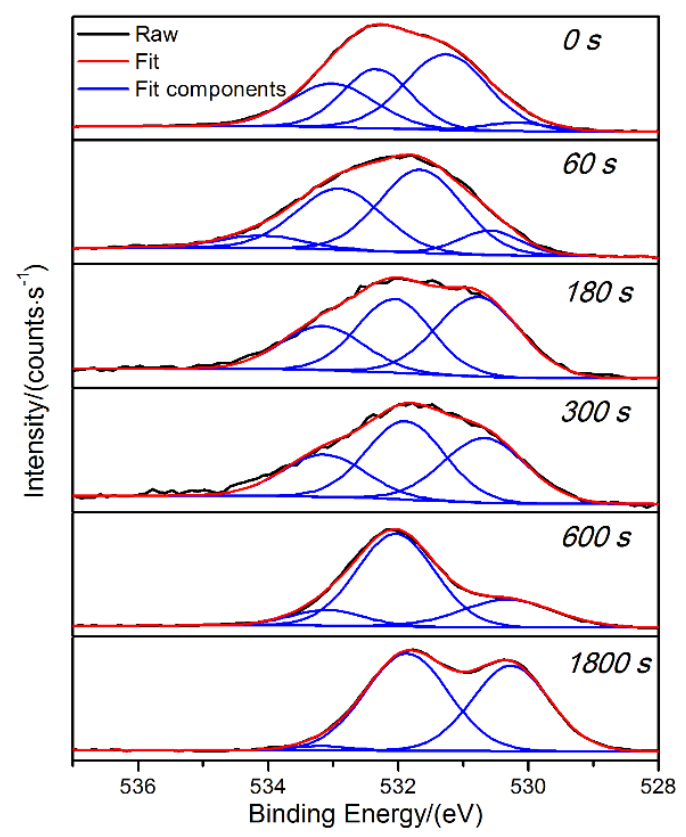

图 S3 不同还原时间复合膜表面的 O1s XPS 图谱, 拟合分峰随结合能增加依次 为: $\mathrm{ZnO}$ 的 $\mathrm{Zn}-\mathrm{O}$ 键、 $\mathrm{GO}$ 羰基 $\mathrm{C}=\mathrm{O}$ 和羧基 $\mathrm{COOH}$ 的混合峰、环氧 $\mathrm{C}-\mathrm{O}-\mathrm{C}$ 键和羟基 $\mathrm{C}-\mathrm{OH}^{\mathrm{s} 1, \mathrm{~s} 2}$ 。注意到与 $0 \mathrm{~s}$ 相比, 60s 图谱中 $\mathrm{C}-\mathrm{OH}$ 分峰相对于 $\mathrm{C}-\mathrm{O}-\mathrm{C}$ 分峰的含量显著减小; 结合 $\mathrm{C} 1 \mathrm{~s}$ 分析中, $60 \mathrm{~s}$ GO 中环氧基含量较 $0 \mathrm{~s}$ GO 显著下降的结论, 可得 $60 \mathrm{~s} \mathrm{GO}$ 中的羟基也大幅减少

Fig.S3 The O1s XPS spectra of the GO-ZnO composite films with different reduction time. The fit components are the $\mathrm{Zn}-\mathrm{O}$ bonds in $\mathrm{ZnO}$, the carbonyl $\mathrm{C}=\mathrm{O}$ and carboxyl $\mathrm{COOH}$ mixed signal, the $\mathrm{C}-\mathrm{O}-\mathrm{C}$ epoxide and the hydroxyl $\mathrm{C}-\mathrm{OH}$ with increasing order of the binding energy ${ }^{\mathrm{S1}, \mathrm{S} 2}$. The relative intensity of the $\mathrm{C}-\mathrm{OH}$ peak to the $\mathrm{C}-\mathrm{O}-\mathrm{C}$ peak of the 60 s sample is smaller than that of the 0 s sample, and the intensity of the $\mathrm{C}-\mathrm{O}-\mathrm{C}$ peak in the $60 \mathrm{~s}$ sample has dropped a lot from that in the 0s sample as is concluded in the $\mathrm{C} 1 \mathrm{~s}$ spectra, thus the content of the $\mathrm{C}-\mathrm{OH}$ bonds in the 60 s sample is less than that in the 0s sample 


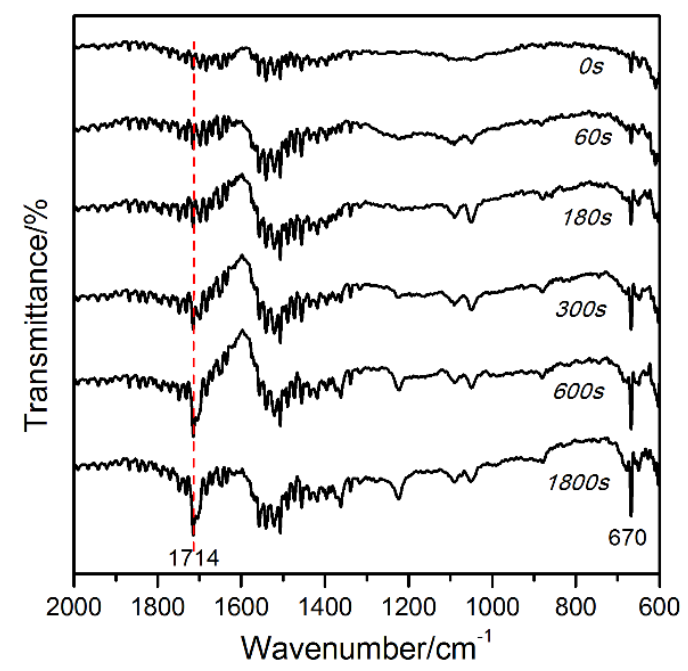

图 S4 各 GO-ZnO 复合膜的 FTIR 衰减全反射透过率图谱

Fig.S4 The FTIR attenuated total reflection transmittance spectra of different GO-ZnO composite films

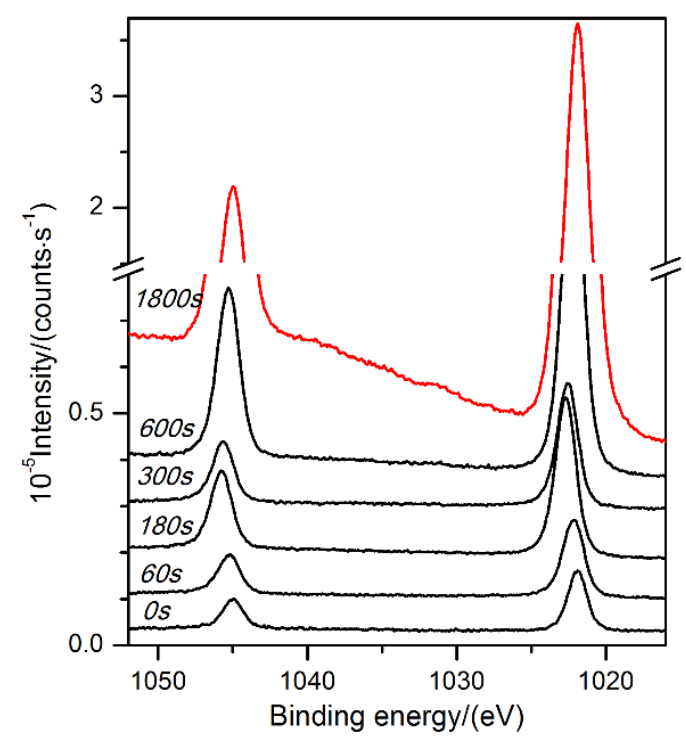

图 S5 不同还原时间复合膜表面的 Zn2pXPS 图谱。随时间变化, 各样品 Zn2p 峰的线形未发生显著变化, 表明 $\mathrm{ZnO}$ 结构在电化学处理过程中一直保持稳定 Fig.S5 The Zn2p XPS spectra of the GO-ZnO composite films with different reduction time. The curved shape remains constant with time, indicating that the chemical structure of $\mathrm{ZnO}$ didn't change during the electrochemical process 


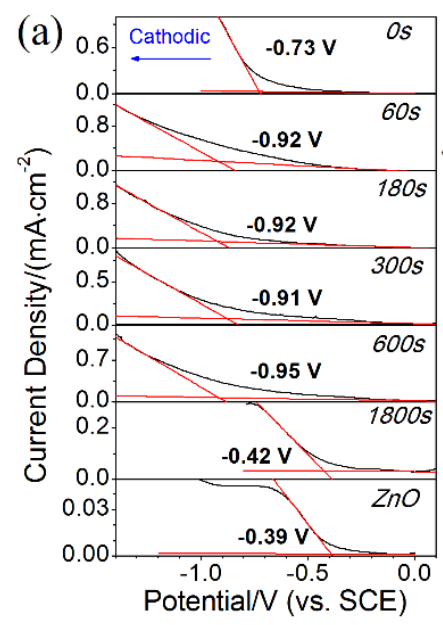

(b)
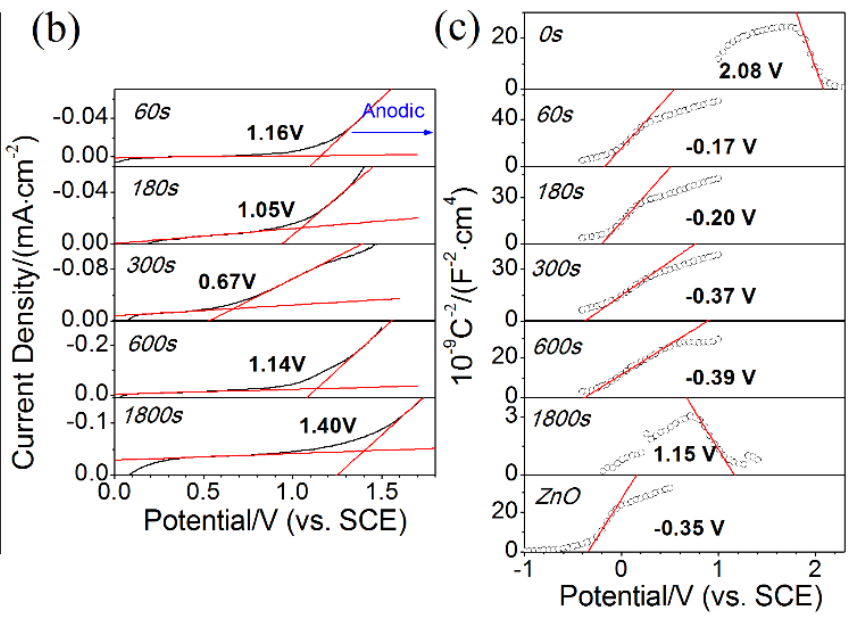

图 S6 样品经过电化学测试得到的(a) 阴极线性扫描伏安(LSV)曲线, (b) 阳极 LSV 曲线和(c) 电化学阻抗(EIS) Mott-Schottky 曲线。测试采用三电极体系, 即 样品薄膜为工作电极, $\mathrm{Pt}$ 片和 SCE 分别为对电极和参比电极, 在 $0.5 \mathrm{~mol} \cdot \mathrm{L}^{-1}$ $\mathrm{Na}_{2} \mathrm{SO}_{4}$ 溶液中进行。LSV 测试的扫描速率为 $5 \mathrm{mV} \cdot \mathrm{s}^{-1}$, EIS 测试的电位增幅为 $0.01 \mathrm{~V}$, 测试频率为 $1000 \mathrm{~Hz}$ 。对 LSV 测试, 阴极扫描和阳极扫描中电流出现 线性增长的起始电位分别与电极表面半导体的导带底(CBM)和价带顶(VBM)位 置对应 ${ }^{S 3, S 4}$ 。其中在进行阳极 LSV 测试时, Os 复合膜和 ZnO 膜在曲线出现激 增前即由于施加了较大的电位造成电极表面水分子氧化生成 $\mathrm{O}_{2}$ 并使电极破坏, 因此未能得到 VBM 值。对 EIS 测试, 曲线线性段切线与横轴的交点值与电极表 面半导体的费米能级对应, 切线斜率则与载流子浓度相关 ${ }^{\mathrm{S}} 4 \mathrm{~S} 5$, 其中斜率符号的 正和负分别表示半导体载流子极性为 $n$ 型和 $p$ 型 $\mathrm{S} 6$

Fig.S6 (a) and (b) are Cathodic and Anodic Linear sweep voltammograms (LSVs) of different samples; (c) Electrochemical impedance spectra (EIS) exhibiting Mott-Schottky characteristics of different samples. The measurements were conducted in $0.5 \mathrm{~mol} \cdot \mathrm{L}^{-1} \mathrm{Na}_{2} \mathrm{SO}_{4}$ solution using a three-electrode configuration with the samples as working electrodes, the $\mathrm{Pt}$

foil and the SCE as the counter electrode and the reference electrode, respectively. The LSVs were measured with a scan rate of $5 \mathrm{mV} \cdot \mathrm{s}^{-1}$, the EIS were measured with an amplitude of $0.01 \mathrm{~V}$ and frequency of $1000 \mathrm{~Hz}$. For the LSVs, the crossings of the extrapolations from the initial stage and the abrupt increase stage in pace with the cathodic and anodic scanning could be approximated as the position of the Conduction Band Minimum (CBM) and 
Valence Band Maximum (VBM) of the semiconductor on the electrode, respectively ${ }^{\mathrm{S3}, \mathrm{S} 4}$. The results didn't give the VBMs of the $0 \mathrm{~s} \mathrm{GO}$ and $\mathrm{ZnO}$, because the oxygen bubbles were electrochemically generated at the surfaces of the electrodes when scanning to more positive potentials, propagating the films breakdown. For the EIS, the Fermi level and doping concentration of the semiconductor could be determined by the zero-crossing and slope of the tangent from the linear region of the Mott-Schottky plot, respectively ${ }^{\mathrm{S4}, \mathrm{S} 5}$, and the slope direction reflects the carrier type with positive as $n$-type and negative as $p$-type ${ }^{\mathrm{s} 6}$

References

(S1) Pradhan, D.; Leung, K. T. Langmuir 2008, 24, 9707. doi: 10.1021/la8008943

(S2) Yu, Y. H.; Lin, Y. Y.; Lin, C. H.; Chan, C. C.; Huang, Y. C. Polym. Chem. 2014, 5, 535. doi: 10.1039/c3py00825h

(S3) Dolai, S.; Dass, A.; Sardar, R. Langmuir 2013, 29, 6187. doi: 10.1021/la401437r

(S4) Yeh, T. F.; Teng, C. Y.; Chen, S. J.; Teng, H. Adv. Mater. 2014, 26, 3297. doi: 10.1002/adma.201305299

(S5) Boix, P. P.; Ajuria, J.; Etxebarria, I.; Pacios, R.; Garcia-Belmonte, G.; Bisquert, J. J. Phys. Chem. Lett. 2011, 2, 407. doi: 10.1002/adma.201305299 (S6) Jang, H.; Kwon, H. J. Electroanal. Chem. 2006, 590, 120. doi: 10.1016/j.jelechem.2006.02.031 